\title{
Vivir (subjetivamente) desde el feminismo en cuanto que mujer. Algunas reflexiones teóricas iniciales
}

To live (subjectively) from a feminist perspective as a woman.

Some initial theoretical reflections

Lía González Estepa·lia.gonzalez@deusto.es

UNIVERSIDAD DE DEUSTO

Raquel Royo Prieto • raquel.royo@deusto.es

UNIVERSIDAD DE DEUSTO

María Silvestre Cabrera • maria.silvestre@deusto.es

UNIVERSIDAD DE DEUSTO

Recibido: $27 / 02 / 2017$

Aceptado: 30/05/2017

\section{Resumen}

El contenido de este artículo se basa en parte del trabajo de reflexión teórica que se está llevando a cabo en torno a un proyecto de investigación que busca aproximarse a los relatos de cuarenta informantes, situadas social y políticamente como mujeres, respecto a su propia experiencia vital como feministas / desde el feminismo. El recorrido teórico exploratorio que se presenta, lejos de ser exhaustivo, responde al resultado de un primer acercamiento mediado por nuestra trayectoria académica e ideológica particular y las inquietudes iniciales que derivan de la misma. Así, los conceptos y planteamientos que recogemos no constituyen para nosotras un marco interpretativo incuestionable, sino un punto de partida sobre el que comenzar a debatir.

Palabras clave: subjetividad feminista; régimen patriarcal; realidades liberadoras-feministas.

\section{Abstract}

The content of this article is based in part on the work of the theoretical reflection that is being carried out around a research project that seeks to approach the accounts of forty informants, socially and politically situated as women, regarding their own life experience as feminists / from a feminist perspective. The theoretical exploratory course that is presented, far from being exhaustive, responds to the result of a first approach mediated by our particular academic and ideological trajectory and the initial concerns that derive from it. Thus, the concepts and proposals that we collect do not constitute for us an unquestionable interpretative framework, but a starting point on which to begin to debate.

Keywords: feminist subjectivity; patriarchal regime: liberating realities-feminist realities. 


\section{INTRODUCCIÓN}

El contenido de este artículo se basa en parte del trabajo de reflexión teórica que se está llevando a cabo en relación a un proyecto de investigación que busca aproximarse a los relatos que presentan cuarenta informantes, situadas social y políticamente como mujeres, en torno a su propia experiencia vital como feministas / desde el feminismo.

El trabajo de reflexión teórica (aún en curso) que se pretende implementar en este sentido no nos gustaría que derivara en una concreción de pautas o indicadores rígidos que encorseten las vivencias de nuestras informantes, sino, más bien, en una guía que nos posibilite acercarnos a las mismas, si bien de manera inevitablemente parcial, también enriquecedora en la medida que podamos ponerlas en diálogo — quizás también en discusión—con diferentes conceptos y aportes teóricos.

Sin embargo, resulta indispensable reconocer que a este trabajo aún le queda mucho camino por recorrer, y que los planteamientos que presentamos a continuación, lejos de constituir para nosotras un marco incuestionable, no son ni más ni menos que un punto de partida, sobre el cual comenzar a debatir.

\section{SER PARA MÍ. EMPODERARME Y EMPODERARNOS, DESDE EL FEMINISMO. EN PALABRAS DE LAGARDE}

¿Qué entendemos por vivir desde el feminismo en cuanto que mujer?

En este momento inicial, para nosotras cabe la posibilidad de entender que vivir desde el feminismo en cuanto que mujer podría aproximarse a lo que Lagarde (2014:109; 2005a:220; 2001:28) llama estar «empoderada», en la medida que ello implica que quienes son aprehendidas, tanto por el conjunto sociocultural que las rodea como por ellas mismas, a través de la categoría mujer vivan desde el "ser-para-mí», reconociendo a su vez legítimo y necesario que todas las demás mujeres también lo hagan (Lagarde, 2001:204).

Para Lagarde (2014; 2005a; 2001), ser-para-mí significa colocarse a una misma en el centro de la vida propia; reconociéndose como sujeta, ciudadana, humana...; valorarse y estimarse desde la autonomía, no en función de la adecuación a intereses ajenos; no subordinar el bienestar y la dignidad individual en pos de otras personas o fines; adueñarse, en suma, del sentido de la existencia que se integra, ponerla en valor, y convertirse en primera responsable de la misma. Según la autora, cuando las mujeres se viven a ellas mismas desde este estatus, se empoderan, y cuando se miran unas a otras autorizándose como legítimas del mismo, empoderan la categoría social y política mujer; lo que, al tiempo, contribuye a respaldar el estatus individual en cada una. 
Lagarde (2014:132) explicita que «ninguna mujer empieza siendo autónoma y libre» (elementos imprescindibles del estar empoderada), de ahí que, para llegar a este estatus, sea necesario desarrollar un proceso consciente de empoderamiento. Pero no cualquiera, sino uno muy concreto y específico, que se arraiga en el mismo centro del sentido de la lucha feminista: el proceso de empoderamiento - feminista - que posibilita que las mujeres dejen de vivir como «ser-para-otros» y pasen a construirse como «ser-para-sí», asumiendo que tanto ellas como todas las demás mujeres son dignas de ello (Lagarde, 2001:202).

De hecho, es De Beauvoir $(2000,1972)$ quien denuncia que las mujeres son históricamente llamadas a alienarse como seres para los otros: objetos, al servicio de hombres-sujetos; y, años más tarde, es el feminismo radical quien, profundizando en esta línea, reivindica la necesidad de acabar con un orden político patriarcal, asentado en el devenir de la historia, que asume a las mujeres en calidad de dominadas en favor del privilegio y del poder de los hombres (Millett, 2000). Tal y como expone Sau (2000), el feminismo, en cuanto que movimiento social y político, se caracteriza por articularse en torno a la toma de conciencia y la activación en favor de la liberación de las mujeres. Es decir, en favor de conseguir que las mujeres vivan no como objetos para..., sino como sujetas libres: seres para ellas mismas. Por tanto, creemos que podría entenderse que cuando una mujer asume que le es legítimo vivir en cuanto que ser para sí, estimando igualmente legítimo que las demás mujeres también lo hagan, vive este proceso de empoderamiento al que hace alusión Lagarde (2014), en consonancia con el sentido de la lucha feminista. ¿Vive desde el feminismo?

A modo de punto de referencia que nos anime a comenzar a indagar, planteamos que, quizás, cabe entender que podría vivir desde el feminismo en este sentido aquella mujer que, ubicada desde su particular posición vital interseccional ${ }^{1}$ (Crenshaw, 1989), consigue distanciarse de la opresión de género y conectarse con realidades alternativas liberadoras-feministas.

\section{LA OPRESIÓN DE GÉNERO: EL RESULTADO DE UN RÉGIMEN POLÍTICO, HABITUADO PERO «ALTERNABLE». DESDE LOS CIMIENTOS DEL FEMINISMO RADICAL. ENTRE BOURDIEU Y BERGER Y LUCKMANN}

Para dar sentido a nuestro mencionado punto de referencia, entendemos que un primer paso imprescindible es concretar qué podríamos asumir como opresión de género.

\footnotetext{
${ }^{1}$ Cabe no pasar por alto que las mujeres pueden vivir sujetas no solo a condicionantes de género, sino también a otros condicionantes sociales, económicos, políticos... (Crenshaw, 1989). Esto es, es posible que sus vidas se vean atravesadas por categorías plurales de desigualdad y discriminación que les dificulten el ejercicio efectivo de la libertad, dentro del marco específico constituido en torno a su experiencia vital concreta (Expósito, 2012).
} 
Siguiendo la tesis eje del feminismo radical de los años sesenta y setenta, liderado por las teorías de Millett (2000) y Firestone (1976)², consideramos que cabe asumir como opresión de género el entramado de relaciones de poder que materializa la distinción de la especie humana en dos tipos, y coloca a uno como dominador del otro, en base a la siguiente premisa ideológica que se arraiga a través de la historia: existe una diferencia sexual de corte natural/biológico que propicia que haya, por un lado, seres de sexo femenino — denominados mujeres - y, por otro, seres de sexo masculino — denominados hombres-; estando dotados cada uno de características y capacidades diferentes y complementarias entre sí, que les lleva a ocupar inherentemente un espacio de poder desigual —de subordinación del ser femenino hacia el masculino- dentro de la estructura sociocultural.

Entendiendo ideología, con Berger y Luckmann (1998:157), como «definición particular de la realidad» ligada a "un interés de poder concreto», cabe remarcar el profundo carácter ideológico de esta premisa que, ya advierte Mill en el siglo xix, sirve para limitar el poder de la mitad de la Humanidad (De Miguel, 1994). Con el objeto de denunciarlo en dichos términos, el feminismo radical acuña el concepto de género ${ }^{3}$, que utiliza para dar nombre al sesgo histórico-sexual identificado como productor de, no solo desigualdad, sino también opresión cultural, social-relacional, psicológica y física (Firestone, 1976). En la misma línea, Millett (2000) populariza también el concepto de patriarcado, que posibilita designar la vigencia de un orden sociocultural político que sustenta y legitima las relaciones de poder reguladas por el género.

Pero, ¿cómo de arraigado puede llegar a estar dicho sistema en la vida de las mujeres?

Bourdieu (2000:53-54, 73) analiza esta cuestión de forma exhaustiva y defiende que la clave fundamental del arraigo es la «dominación simbólica», que se enraíza en el «hábito», de forma subterránea a la conciencia, a través del «inconsciente histórico».

Según este autor, el género puede operar directamente mediante sensaciones percibidas como instintivas, sin necesidad de haber sido directamente legitimado antes por la razón. Explica que en la medida en que tanto la persona individual como la sociedad en su conjunto se construyen dentro de un medio de tradición histórico-estructural impregnado por el patriarcado, el arraigo de la opresión de género no se produce entrando desde fuera, sino saliendo desde dentro. De esta forma, el género (y su poder de opresión) puede instaurarse en valores y prácticas humanas de manera imperceptible, sin levantar sospecha, debido a que se encuentra habituado dentro del sentido común, constituido, en palabras

\footnotetext{
2 Aunque entendemos que Millett (2000) y Firestone (1976) coinciden en este planteamiento que exponemos, cabe puntualizar que el desarrollo teórico que cada autora lleva a cabo es propio y diferente; sin embargo, escapa de nuestro objeto ahondar en este campo.

3 Como explica Lamas (1997:102), una de las pioneras en trabajar la cuestión del género desnaturalizando las diferencias entre mujeres y hombres es la antropóloga Mead, que se erige como una importante referente; sin embargo, es el feminismo radical quién posteriormente marca un crucial punto de inflexión al denunciar que tales diferencias pasan a constituirse dentro del orden social como formas de legitimación de la situación de opresión de las mujeres.
} 
de Bourdieu (2000:49), por el «consenso práctico dóxico» de los sistemas de pensamiento. El nombre que da Bourdieu (2000:51) a esta forma de dominación, tan invisible como profunda y poderosa, es «violencia simbólica».

Yendo al ámbito de la persona individual, la teoría del hábito de Bourdieu (2000) permite entender que, al transgredir un mandato de género, es posible que sea el mismo cuerpo el primero que castigue (aunque no el único), provocando manifestaciones físicas involuntarias que delaten la incomodidad interna. Y también puede ocurrir que el arraigo opere, no castigando, sino atrayendo, provocando deseo, agrado o placer en relación a evidencias y pautas de género. El autor explica que los efectos de sujeción hacia el género pueden darse tanto en mujeres como en hombres, pues, el hecho de que este sistema de relaciones de poder coloque a unas como dominadas y a otros como dominadores no implica que exista libertad efectiva en alguno de los dos espacios. El género, en tanto en cuanto estipula el deber ser de la persona, constriñe y censura las opciones vitales en cualquiera de los casos - aunque de forma desigual-.

En relación al arraigo de la opresión de género que pueda darse específicamente en las mujeres, conviene recordar que De Beauvoir (1972) sienta las bases para la transformación de las tesis feministas al defender que la dominación no solo constituye una injusticia impuesta a las mujeres desde fuera (por los hombres, en concreto, o el contexto sociocultural, en general), sino que forma parte del foro interno de ellas mismas. En consecuencia, las mujeres viven desde la posición subordinada y alienada de objeto, en relación al hombre sujeto, no solo en pos de conseguir aprobación y agrado externo, sino también interno. De Beauvoir (2000) analiza cómo el conocimiento humano (filosófico, científico...) se ha desarrollado tomando como medida universal la perspectiva del hombre, de tal modo que la mujer solo puede ser concebida, incluso por ella misma, como excepcional y siempre en relación a su papel dentro del mundo de él; lo que, como hemos mencionado anteriormente, la lleva a vivirse en calidad de ser para... Remarcamos que este hecho implica una forma de vivir, con motivo de no dejar pasar que entendemos que interpela a la integridad de la persona, abarcando tanto su pensar, su sentir...; que no consideramos en ningún caso compartimentos estancos, sino esferas estrechamente interrelacionadas e interdependientes (Gilligan, 1994; Hochchild, 1990).

Pero estar ubicada como dominada, bajo la opresión de género, no significa permanecer necesariamente estática o pasiva. De Beauvoir (1972) se muestra contundente al afirmar que, bajo la opresión de género, las mujeres, a la par que son devaluadas, también aprenden a tejer formas de uso y abuso de poder ocultas. Lagarde (2005b; 2001) profundiza igualmente en esta idea al defender que las mujeres, desde la opresión de género -en cautiverio ${ }^{4}$ - , aprenden a sobrevivir de manera creativa, en función de su situación dentro otras relaciones de poder articuladas por cuestión de clase social, etnia... donde puede

${ }^{4}$ Lagarde (2005b:155) crea la categoría «cautiverio» con objeto de constituir la expresión político-cultural que defina la condición vital marco de las mujeres dentro del mundo patriarcal. 
darse el caso de que se ubiquen como dominadoras ${ }^{5}$, pero sin conseguir por ello un estatus vital de mayor libertad genérica.

En este sentido, Bourdieu (2000:69) explica que muchas mujeres se hacen expertas en conseguir sus metas a través de aprender a actuar en la sombra, tendiendo a fundamentar su éxito en herramientas conspirativas y sutiles. Pero, como subraya este autor, dichas vías de control y poder no dejan de ser «armas de la debilidad», en la medida que bien los medios y bien los objetivos finales que las guían sigan estando articulados en base a lógicas de género. A modo de ejemplo, Bourdieu (2000:48) hace alusión a lo que llama el «amor posesivo de los poseídos» que puede identificarse, entre otros, en los casos en que se hace uso de diferentes formas de chantaje emocional para mantener la vinculación de un ser querido, sin cuestionar el hecho de que ese deseo de vínculo pueda ocultar una sustancial situación de dependencia hacia tal relación afectiva.

En suma, según lo expuesto, la opresión de género relega a la mujer a una posición vital subordinada; sin embargo, esta puede aprender a acomodarse dentro de dicha subordinación. Pero, a pesar de tal capacidad, la mujer seguirá estando en última instancia dominada, pues, en tanto en cuanto interioriza las bases ideológicas de género, la deriva de sus deseos de incidencia la llevará a reforzar el sistema de ordenación patriarcal que la somete.

Aún con todo esto, enlazando con las críticas que desde la teoría feminista Adkins (2004) realiza a Bourdieu (2000), creemos que la opresión de género no es inevitable, y que, si bien la teoría del hábito parece dejar poco margen para ello, cabe pensar que las personas individuales tienen capacidad y opción de distanciarse de ella y alternarla por otras realidades. Fundamentamos tal afirmación en los siguientes contenidos de la teoría de la construcción social de la realidad desarrollada por Berger y Luckmann (1998) ${ }^{6}$.

En base a estos autores, entendemos que todo orden social — sociedad — se construye a través de un «proceso dialéctico» compuesto por tres momentos: «externalización» (1), necesidad humana de generar actividad en relación con el entorno; «objetivación» (2), asimilación del tipo de actividad generada como opción a disposición del común, es decir, realidad

\footnotetext{
${ }^{5}$ En este sentido, es interesante reparar en la oportunidad que ofrece la perspectiva interseccional para identificar no solo condiciones de opresión, sino también de privilegio (Platero, 2012).

${ }^{6}$ Aunque aquí nos basamos en la teoría desarrollada por Berger y Luckmann (1998), conviene mencionar que, como explica Royo (2011), la idea orginal que motiva los fundamentos de dicha teoría tiene como germen las tesis planteadas anteriormente por Schütz, a las que no acudimos directamente para la elaboración de este artículo. Por otro lado, también conviene explicitar que somos conscientes de que este campo de estudio, que debate la posibilidad o no de cambio y transformación, cuenta con un largo recorrido dentro de la historia de la Sociología, especialmente, en relación a los conceptos de socialización y desviación, abordados desde diversos enfoques. Sin embargo, en este momento de nuestro recorrido teórico particular, tomamos la decisión de ceñirnos a las aportaciones de Berger y Luckmann (1998), considerando que hacerlo, aunque inevitablemente deja fuera múltiples y enriquecedoras opciones interpretativas, nos da herramientas teóricas clave para empezar a andar, a la luz de un enfoque sociológico capaz de advertir tanto coacción, represión, dominación..., como transgresión, cambio, transformación..., desde una perspectiva relativamente sencilla, significativamente clara, y, en suma, entendida como accesible e idónea en relación al conjunto de posibilidades y capacidades con las que contamos actualmente.
} 
objetiva; e «internalización» (3), aprehensión de un acontecimiento como dotado de significado específico en función de lo ya establecido por el contexto (Berger y Luckmann, 1998:164). Este proceso tiene como elemento eje el lenguaje, que sirve para abstraer la experiencia individual y convertirla en posibilidad objetiva al alcance del común (Berger y Luckmann, 1998:92). De este modo, se logra acotar las opciones, crear pautas fijas..., conformar «instituciones sociales», y, en consecuencia, generar una sensación psicológica de estabilidad y alivio que evita caer en la angustia ante la inmensidad (Berger y Luckmann, 1998:76). En esta línea, Berger y Luckmann (1998:106-107) exponen el fenómeno de la «reificación», que supone el olvido de que lo que se entiende por realidad objetiva es una construcción humana (una opción entre las posibles) y el paso a asumir la misma como verdad independiente. Tal fenómeno limita la posibilidad de duda ante las realidades objetivadas y convertidas en instituciones sociales, que se sustenta en el tiempo a través de procesos de legitimación y control social (Berger y Luckmann, 1998: 120-121, 77).

Pero los autores también sostienen que el orden social se produce en diálogo permanente con las personas individuales, que no son receptoras pasivas. Afirman que, si bien resulta imprescindible contar con una realidad objetiva de referencia, la persona individual nunca asume de manera absoluta lo que le es dado, pues desarrolla capacidad de disonancia subjetiva. Así, las personas pueden dudar, imaginar alternativas y, por ende, «transformar» o «alternar» su realidad (Berger y Luckmann, 1998: 196). ¿Qué precisan para ello? Berger y Luckmann (1998: 135, 204-215) exponen como fundamental aprehender un universo simbólico alternativo y contar con respaldo social.

Por un lado, aprehender un universo simbólico alternativo - es decir, un marco de significados diferente al institucionalizado - resulta clave en la medida en que si se carece de él, aunque pueda articularse cierto escepticismo ante el orden social establecido, no será posible salir de este, pues no hay alternativa alguna donde habitar. Y es que Berger y Luckmann (1998:135) mantienen que el ser humano "vive dentro de universos simbólicos». Por otro, el respaldo social se vuelve también imprescindible debido a que la persona no puede sostener por sí sola una realidad, necesita como mínimo el reconocimiento de su gente próxima —en palabras de Berger y Luckmann (1998:189), sus «otros significantes»-, claro está que la persona puede tejer nuevas relaciones interpersonales con este fin. Cuando la realidad alternativa la comparte una minoría, esta puede ser excluida del orden social o bien asimilada en cuanto que tal sin resultar amenazante; cuando la realidad alternativa consigue atraer a una mayoría social significativa, sin embargo, peligra el status quo de la realidad institucionalizada en cuestión: se abre la posibilidad de que esta sea transformada o alternada por otra (Berger y Luckmann, 1998: 204-215).

En consonancia con estas bases teóricas, creemos que la opresión de género podría comprenderse como una opción de interrelación humana, objetivada y naturalizada por el fenómeno de la reificación en el devenir de la historia, institucionalizando así el régimen del patriarcado, que mantiene su status quo a través de mecanismos de legitimación y control social. 
Igualmente, consideramos que cabe entender que la persona individual internaliza en mayor o menor medida la opresión de género en función de lo presente que esta esté en su proceso de socialización; al tiempo que esta misma persona podría distanciarse de la opresión de género en la medida que desarrolle capacidad subjetiva para ponerla en duda, pero solo conseguirá vivir fuera de esta realidad si obtiene un marco de significados alternativo y un entorno social que legitime el mismo.

En relación a la capacidad subjetiva de disonancia, creemos interesante traer a colación la propuesta de Woolf (2008), que, a principios del siglo xx, apunta la suficiencia económica y la disposición de «una habitación propia» (independiente al resto del hogar y sus habitantes) como condiciones indispensables para que las mujeres puedan desarrollar su pensamiento y creatividad. Woolf (2008) advierte que la persona precisa para desarrollar su potencial de una situación vital que le conceda tiempo y espacio para sí; lo que, según la novelista, implica autonomía y dedicación a una misma, que es precisamente aquello que históricamente se les ha negado a las mujeres. Siguiendo a Chodorow (2003), Gilligan (1994) y Hochchild (1990), no olvidamos en este marco la importancia del potencial de las mujeres, no solo en cuanto a pensamiento racional, sino también en cuanto a sentimiento; y recordamos igualmente a Connell (2003), que defiende que el cuerpo no es un escenario pasivo y, de hecho, puede incitar a la persona a dudar de lo establecido, a través de experimentar sensaciones disonantes con la norma (de agrado hacia lo prohibido o rechazo hacia lo normalizado) 7 . En cualquier caso, referir que entendemos que el desarrollo del sentimiento y la sensibilidad corporal tampoco pueden producirse cuando se carece de tiempo y espacio propio para dedicarse a una misma.

Entrado el siglo xxI, Zafra (2010) reactualiza este concepto de habitación propia acuñado por Woolf (2008) y habla de «un cuarto propio conectado» a internet —y, por ende, al mundomás potente que la primera habitación, debido a que este permite a las mujeres disfrutar del espacio para sí sin estar aisladas, sino conectadas con infinidad de realidades que pueden aprehenderse de forma ordenada y selectiva desde la comodidad, privacidad y, en suma, protección de la intimidad de cada una. La idea de que la capacidad de conexión con el exterior potencia las opciones de desarrollo — con independencia de que tal conexión se produzca o no a través de internet- puede enlazarse a la premisa, anteriormente expuesta, de que para vivir desde otras realidades es preciso vincularse con..., pues no es una empresa posible en soledad. Lagarde (2001:819) también coincide en este punto, indicando que una mujer que solo rechaza la opresión de género es una mujer "fallida», abocada a la frustración; y, del mismo modo, defiende que este tipo de mujer es todavía proclive a seguir reproduciendo dinámicas acordes a la norma patriarcal, aún sin quererlo y sin saberlo, porque no cuenta con herramientas para dejar de hacerlo; así, según la autora, quizás pueda procurarse cierta independencia en relación a la opresión, pero nunca autonomíå.

\footnotetext{
${ }^{7}$ Esteban (2013) en «Antropología del cuerpo. Género, itinerarios corporales, identidad y cambio» profundiza en el estudio de esta importancia del cuerpo dentro del marco de la producción, reproducción y también transformación de las relaciones de poder de género.

${ }^{8}$ Esta idea enlaza con los postulados de Foucault $(2012,1987)$, que defienden que no es posible resistir al poder a través de la separación o el alejamiento, pues no existe de por sí espacio de libertad alguno.
} 
Por tanto, según lo referido, estimamos posible plantear que, quizás, las mujeres, socializadas bajo el patriarcado, tengan opción de distanciarse de la opresión de género siempre y cuando cuenten con capacidad subjetiva de disonancia, condiciones de tiempo y espacio para dedicarse a ellas mismas y contacto con realidades alternativas y, además, estas realidades estén sostenidas por un conjunto social de algún modo significativo para ellas.

Resulta pertinente aclarar que el distanciamiento hacia la opresión de género en torno al que pensamos tendría que ver con el modo en que la persona entiende el mundo que la rodea, pero no necesariamente con la modificación del mismo; esto es, consideramos que podría distanciarse de la opresión de género aquella mujer que, siguiendo la conocida metáfora que populariza Lienas (2007), se pone las "gafas violeta» del feminismo y deja de asimilar el sistema patriarcal como verdad natural y legítima; independientemente de que consiga o no dejar de vivir afectada —oprimida — por él.

De igual forma, puntualizamos que, según los términos planteados, para afirmar que las mujeres viven desde el feminismo no bastaría con que estas articularan cualquier tipo de distanciamiento en relación a la opresión de género: las realidades alternativas que permitieran el distanciamiento deberían constituir, imprescindiblemente, realidades liberadoras-feministas; es decir, realidades acondicionadas para que las mujeres se aprehendan como seres para ellas mismas.

\section{LAS REALIDADES ALTERNATIVAS LIBERADORAS- FEMINISTAS. ¿CÓMO IDENTIFICAR REALIDADES SUSCEPTIBLES A LA LIBERACIÓN? UNA APROXIMACIÓN BASADA EN PLANTEAMIENTOS DE FRASER}

Siguiendo los planteamientos teóricos expuestos hasta el momento, creemos que puede entenderse que tomar distancia de la opresión de género no convierte automáticamente a las mujeres en seres para ellas mismas, porque es preciso que primero estas se construyan como tales; y cabe inferir que para ello resulta imprescindible que la realidad alternativa con la que se conecten así lo posibilite. Pero ¿cuándo podría entenderse que una realidad está en condiciones de articular dicha posibilidad?

Conscientes de que podrían desarrollarse múltiples vías teóricas para responder a esta pregunta, optamos por comenzar reparando en algunos de los planteamientos de Fraser $(2011,2008)$ en torno a la interpretación de las necesidades humanas y la justicia.

El primer planteamiento que consideramos especialmente interesante tiene que ver con preguntarse si la realidad en cuestión es o no capaz de adaptarse a lo que, según Fraser (2011), constituye una interpretación justificable de las necesidades humanas. Dado que Fraser (2011) expone la interpretación de las necesidades humanas como forma de enten- 
der e interpretar un acontecimiento social x, creemos posible utilizar sus parámetros también a la hora de analizar aquello que, siguiendo a Berger y Luckmann (1998), hemos llamado aquí realidades; pues, de hecho, ambos conceptos tienen mucho en común: tanto uno como otro se refieren a una opción interpretativa que busca ser objetivada por el conjunto social.

Según la propuesta de Fraser (2011), es necesario considerar tanto el procedimiento de construcción de la interpretación como sus consecuencias. En cuanto al procedimiento, refiere que podrán asumirse como justificables aquellas interpretaciones construidas a través de procesos comunicativos afines a las ideas de democracia, igualdad y justicia; lo que para la autora implica analizar la prevalencia o no de criterios de inclusión frente a exclusión y de igualdad frente a jerarquía. En cuanto a las consecuencias, explica que pueden asumirse como justificables aquellas interpretaciones tendentes a no generar desventajas entre unos y otros grupos sociales; esto es, aquellas que cuestionen (y rechacen) pautas de dominación y subordinación (Fraser, 2011).

A nuestro juicio, tener en cuenta estas consideraciones puede ayudar significativamente a identificar todas aquellas realidades que pudieran volver a instalar a las mujeres dentro de dinámicas relacionales de opresión - ya sea como dominadas o como dominadoras-. Por una parte, las consideraciones en torno a las consecuencias nos resultan clave de cara a identificar si las realidades son o no, en términos de la autora, transformadoras (Fraser, 2011; 2008). Es decir; si ponen en cuestión y enfrentan la raíz misma de la injustica: la validación de la asimetría de estatus de dignidad entre personas humanas.

Las consideraciones en torno al procedimiento, por otra parte, nos son de especial utilidad para descartar todas aquellas realidades que, si bien abogan por la consecución de igualdad, democracia, justicia..., se construyen sobre dinámicas comunicativas entre los diferentes agentes implicados que poco tienen que ver con dichos principios, obviándose los intereses y condicionantes de unos en favor de los de otros.

En segundo lugar, y de cara a empezar a explorar si la realidad en cuestión puede, efectivamente, garantizar la afirmación de las mujeres en cuanto que seres para ellas mismas, miramos hacia los conceptos de «representación», «reconocimiento» y «redistribución» que aporta Fraser (2008:89-42) ${ }^{9}$.

Fraser (2008:89-42) defiende que la participación política plena, articulada desde el principio de la justicia, requiere de tres elementos: «representación», que implica inclusión de la persona dentro del marco de convivencia como agente que participa del mismo; «reconocimiento», que implica que la persona no se vea impedida por «jerarquías institucionales

\footnotetext{
9 Tomar como referencia estos conceptos de Fraser (2008) no es en absoluto una opción novedosa u original. Cabe citar, por ejemplo, el caso de los aportes teóricos de Esteban (2011) en torno a la crítica del pensamiento amoroso, donde la autora adapta y trabaja los conceptos de reconocimiento y redistribución.
} 
de valor cultural» que la minusvaloren como participante; y «distribución» justa, que implica que la persona no se vea impedida por «estructuras económicas» que le impidan el acceso a recursos necesarios para participar en igualdad de condiciones.

Teniendo a Fraser (2008) como referente, consideramos posible entender que para que las mujeres puedan aprehenderse como seres para ellas mismas es indispensable que estas, en consonancia con la máxima del feminismo radical, se asuman como seres políticas, con capacidad de participar en la gestión de las relaciones de convivencia en cuanto que libres e iguales; y que estimen legítimo reclamar, por tanto, su derecho a estar representadas, a ser reconocidas y a una distribución económica de los recursos que no las deje fuera o en inferioridad de condiciones.

Conviene remarcar que la asunción política requeriría inevitablemente dejar de entenderse desde lo particular y pasar a aprehenderse como parte de un todo dentro del conjunto social (Arendt, 1997). ¿Cuál podría ser la vía para desarrollar tal conciencia política?

Lagarde (2014; 2005a; 2005b; 2001), en la línea de lo ya mencionado anteriormente, habla de resignificar positivamente la categoría mujer(es), mediante el reconocimiento como iguales en la diversidad, la construcción de una memoria histórica de género común que sirva como marco de referencia y amparo... En suma, a través de fortalecerse conectándose y legitimándose unas a otras, y cada una a sí misma, en cuanto que mujeres y, por ende, fortalecer también la categoría en sí, que sirve de plataforma para todas.

No obstante, existen igualmente muchas más. Una de las radicalmente más distintas a esta de Lagarde (2014, 2005a; 2005b; 2001) que hemos mencionado quizás sea la de Butler (2007), que (según nosotras la entendemos) propone romper con la política de identidades fijas, planteando no construir nuevas opciones identitarias estables en sí, sino dejar la puerta abierta a que cada cual pueda servirse de todas las posibilidades, sin excepción, y tenga la libertad de escoger en cada momento. Butler (2007:288) enfatiza que la «deconstrucción de la identidad no es la deconstrucción de la política»; y creemos posible entender que considera factible no partir de crear unas etiquetas fijas de identificación comunes, primero, y articular una política que defienda sus intereses, después, sino crear una nueva forma de política que ampare la libertad de las personas para adherirse, sin censuras, a las pautas que estimen más convenientes en cada momento vital e ir desarrollándose a través de las mismas.

Conscientes de que cabría profundizar en el estudio de la deconstrucción y la construcción de las identidades, en este análisis nos atrevemos a presentar esta breve referencia al pensamiento de Butler (2007), a modo de contrapunto, con la intención de clarificar que en ningún caso queremos plantear que entendamos que construirse como ser para sí deba ligarse a patrones fijos o cerrados de realidad e identidad política. Y es que compartimos con Herrera (2015:5) que no existe una realidad alternativa susceptible de constituirse como espacio liberador-feminista, sino muchas «posibles» y, probablemente, como sostiene esta autora, «necesarias». 
Queremos remarcar así que con los planteamientos de Fraser $(2008,2011)$ que recogemos no buscamos en ningún caso dar con un modelo de realidad único al que adaptarse en pos de la liberación-feminista, sino imaginar un primer marco de condiciones a nuestro entender de utilidad para valorar si tal objetivo puede verse conculcado o no dentro de cualquiera que sea la realidad y la forma de identidad por la que se opte.

Así, siguiendo a Fraser (2011, 2008), consideramos que, a priori, podrían ser susceptibles de constituirse como realidades liberadores-feministas — capaces de posibilitar que quienes han sido socializadas como mujeres se construyan como seres para ellas mismas-, aquellas realidades alternativas: 1) que se produzcan en pos y a través de dinámicas relacionales incluyentes e igualitarias, afines a los principios de igualdad, justicia y democracia; y 2) que promuevan la conciencia política de las mujeres -independientemente de que ello implique o no seguir identificándose con esta categoría- como sujetas legítimas de plena representación, reconocimiento y disposición de los recursos económicos suficientes para interactuar socialmente como seres políticas, libres e iguales.

Conviene aclarar que cuando nos referimos a conectarse con espacios liberadores no estamos suponiendo en ningún caso que el mundo integral de la persona se mueva dentro de este marco; si así fuera, entendemos que no estaríamos hablando solo de la capacidad de las mujeres para aprehenderse como legítimas de..., sino también de la constitución efectiva de estas como personas libres e iguales en la práctica dentro del conjunto social. Esto es, estaríamos hablando de habitar en una sociedad que, en términos de Berger y Luckmann (1998), ha des-institucionalizado el patriarcado y lo ha alternado por una realidad liberadora - feminista- No es a esto a lo que buscamos referirnos. A lo que nos queremos referir es a la puesta en contacto con realidades alternativas, no arraigadas en el conjunto del orden social, pero suficientemente desarrolladas simbólica y socialmente como para poder erigirse a modo de opción real de entender el mundo y la propia existencia de forma divergente a lo establecido por el patriarcado.

\section{CONCLUSIÓN}

Llegado a este punto de nuestra exploración teórica inicial, creemos que, recapitulando, es posible recoger lo siguiente.

Por un lado, que partimos de entender que desarrollar subjetividad feminista, en el caso de aquellas personas situadas desde la categoría social y política mujer, queda atravesado por un proceso de afirmación política que tiene que ver con dejar de asumirse como ser al servicio de otras personas o fines y pasar a construirse como sujeta política (libre e igual).

Por otro, que este proceso de afirmación política puede entenderse como condicionado, entre otros factores, por: 
- La capacidad subjetiva de disonancia de la persona individual respecto a la realidad objetiva-institucional.

- El tiempo y espacio que esta persona pueda dedicar y dedique a trabajar la relación consigo misma, y, así, desarrollar su capacidad subjetiva. El tiempo que dedique a atender su propio cuerpo, su sentimiento, su pensamiento...

- La puesta o no en contacto con lo que aquí hemos entendido como realidades liberadoras-feministas, producidas en pos y a través de dinámicas incluyentes e igualitarias, que posibiliten su afirmación como sujeta política legítima de plena representación, pleno reconocimiento y disposición de recursos económicos suficientes para poder interactuar en cuanto que libre e igual.

— La asimilación o no del universo simbólico de estas realidades.

- Contar o no con un conjunto social significativo para ella que comparta igualmente estas realidades.

No cabe duda de que las cuestiones a matizar, repensar y debatir son innumerables. Y, así mismo, que el recorrido de exploración teórica que se presenta, lejos de constituir un marco exhaustivo y acabado, responde al resultado de un primer acercamiento mediado por nuestra trayectoria académica e ideológica particular y las inquietudes iniciales que derivan de la misma.

De aquí a un tiempo, quizás, parte importante de lo que ahora sentimos como apoyo teórico fundamental deje de resultarnos central o, incluso, cabe la posibilidad de que lo sustituyamos por otras referencias.

A día de hoy, sin embargo, tanto las aportaciones específicas de Lagarde (2014; 2005a; 2005b; 2001), como las bases teóricas del feminismo radical y de De Beauvoir (2000, 1972), nos permiten explorar un marco de interpretación general de las experiencias vitales que relatan nuestras informantes, dentro del cual es posible discernir qué entender como vivencia subjetiva conectada con el feminismo y qué no (cuestión indispensable para nuestro estudio), atendiendo a si esta se produce desde la afirmación de la mujer como sujeta política - libre e igual-o si, por el contrario, se asume como susceptible de subordinación a otras personas o fines.

Decantarnos por apoyarnos en una mirada sociológica a caballo entre la teoría del hábito de Bourdieu (2000) y los fundamentos presentados por Berger y Luckmann (1998) nos permite obtener una vía para comenzar a interpretar la manera en que nuestras informantes gestionan su vida social, asumiendo y rechazando las diferentes formas de interacción que estiman tener disponibles.

La sensibilidad que Woolf (2008) nos ofrece nos anima a focalizar el interés en aspectos de los relatos de nuestras informantes relacionados con la búsqueda y construcción de espacios por y para ellas mismas que de otro modo, a día de hoy, quizás, pasaríamos por alto. 
Y, finalmente, servirnos del sentido de algunos de los planteamientos formulados por Fraser (2011, 2008), creemos que puede resultarnos de gran utilidad para aterrizar nuestro marco de interpretación general dentro de un conjunto concreto de condiciones que especifica qué podríamos entender como indispensable para asumir que, efectivamente, el relato de nuestras informantes denota que estas se legitiman como sujetas políticas - libres e iguales-, rechazando tanto subordinarse a otras personas o fines como adherirse a cualquier forma de dinámica de interacción opresiva, ya sea situándose como dominadas o como dominadoras.

\section{REFERENCIAS BIBLIOGRÁFICAS}

Adkins, L. (2004). Introduction: Feminism, Bourdieu and after. En Adkins y Beverley (eds.) Feminism after Bourdieu (pp. 3-18). Norwich: Blackwell Publishing/The Sociological Review.

Arendt, H. (1997). ¿Qué es la política?. Barcelona: I.C.E. de la Universidad Autónoma de Barcelona.

Berger, P. y Luckmann, T. (1998). La construcción social de la realidad. Buenos Aires: Amorrotu editores.

Bourdieu, P. (2000). La dominación masculina. Madrid: Anagrama.

Butler, J. (2007). El género en disputa: el feminismo y la subversión de la identidad. Madrid: Paidós Ibérica.

Chodorow, N. (2003). El poder de los sentimientos. Buenos Aires: Paidós.

Connell, R.W. (2003). Masculinidades. México: Universidad Autónoma de México.

Crenshaw. K. (1989). Demarginalising the intersection of Race and Sex: a Black Feminist Critique of Antidiscrimination Doctrine. Feminist Theory and Antiracist Politics, University of Chicago Legal Forum, pp.139-167.

De Beauvoir, S. (1972). El Segundo sexo. La experiencia vivida. Vol. II. Buenos Aires: Siglo XXI.

—(2000). El segundo sexo. Los hechos y los mitos. Vol. I, Madrid: Cátedra.

De Miguel, A. (1994). Cómo leer a John Stuart Mill. Madrid: Ediciones Jucar. 
Esteban, M.L. (2011). Crítica del pensamiento amoroso. Barcelona: Edicions Bellaterra.

- (2013). Antropología del cuerpo. Género, itinerarios corporales, identidad y cambio. Barcelona: Ediciones Bellaterra.

Exposito, C. (2012). ¿Qué es la interseccionalidad? Aproximación al tratamiento de la diversidad desde la perspectiva de género en España. Investigaciones Feministas, vol. 3, pp. 203-222.

Firestone, S. (1976). La dialéctica del sexo. Barcelona: Kairós.

Foucault, M. (2012). Vigilar y castigar. Nacimiento de la prisión. Madrid: Biblioteca Nueva. Siglo veintiuno editores.

— (2016). Historia de la sexualidad. 1. La voluntad de saber. México: Siglo XXI.

Fraser, N. (2008). Escalas de justicia. Barcelona: Herder.

- (2011). La lucha por las necesidades: esbozo de una teoría crítica socialista-feminista de la cultura política del capitalismo tardío. En Fraser, N. et al. (eds.), Dilemas de la justicia en el siglo xxI. Género y globalización (pp. 97-138). Illes Balears: Ediciones UIB.

Gilligan, C. (1994). La moral y la teoría psicológica del desarrollo femenino. México: FCE.

Herrera, C. (2015). La construcción sociocultural de la realidad desde una perspectiva Queer. Haika ediciones (on line).

Hochchild, A. R. (1990). The Commercialization of Intimate Life. Notes from Home and Work. Berkeley: University of California Press.

Lagarde, M. (2014). El feminismo en mi vida. Hitos, claves y topías. Madrid: horas y Horas.

—(2005a). Para mis socias de la vida. Madrid: horas y Horas.

- (2005b). Los cautiverios de las mujeres: madresposas, monjas, putas, presas y locas. México: Universidad Autónoma de México.

- (2001). Claves feministas para la autoestima de las mujeres. Madrid: horas y Horas.

Lamas, M. (1997). El género: la construcción cultural de la diferencia sexual. México: Universidad Nacional Autónoma de México. 
Vivir (subjetivamente) desde el feminismo... | Lía González Estepa, Raquel Royo Prieto, M. $\underline{a}$ Silvestre Cabrera

Lienas, G. (2007). El diario violeta de Carlota. Buenos Aires: El Aleph.

Millett, K. (2000). Sexual Politics. United States of America: University of Illinois.

Platero, R. (L.) (2012). Introducción. La interseccionalidad como herramienta de estudio de la sexualidad. En Platero, R. (L.) (ed.) Intersecciones: cuerpos y sexualidades en la encrucijada. Temas contemporáneos (pp. 15-72). Barcelona: Bellaterra.

Royo, R. (2011). El trabajo familiar en la CAPV. Una perspectiva cualitativa en parejas de doble ingreso (Tesis Doctoral). Bilbao: Universidad de Deusto.

Sau, V. (2000). Diccionario ideológico feminista. Vol. I. Barcelona: Icaria.

Woolf, V. (2008). Una habitación propia. Barcelona: Seix Barral, S.A.

Zafra, R. (2010).Un cuarto propio conectado. (Ciber)espacio y (auto)gestión del yo. Madrid: Fórcola. 